\title{
RATES OF REACTIONS IN Cu(II)-BENZOYLACETONE SYSTEM IN AQUEOUS PHASE
}

\author{
MAKOTO HARADA, MASATERU MORI, MOTONARI ADACHI \\ AND WATARU EGUCHI \\ Institute of Atomic Energy, Kyoto University, Uji 611
}

The mechanism and rates of aqueous-phase reactions in cupric ion and benzoylacetone system were elucidated. The exchange reaction between enol and keto forms was expressed by

$$
\mathrm{HE} \stackrel{k_{E}}{\rightleftharpoons} \mathrm{H}^{+}+\mathrm{B}^{-} \stackrel{k_{K}}{=} \mathrm{HK}
$$

The rate constants for enol and keto forms were determined as $k_{E}=81 \mathrm{~s}^{-1}$ and $k_{K}=0.018 \mathrm{~s}^{-1}$, respectively. The reaction rate between enolate ion and cupric ion was well interpreted by Eigen's mechanism and the rate constant of second-order reaction was $k_{C B}=1.1 \times 10^{9} \mathrm{dm}^{3} / \mathrm{mol} \cdot \mathrm{s}$. Direct reactions between cupric ion and the enol and keto forms of benzoylacetone were observed. The rate constants for the enol and the keto forms were 2000 and $12 \mathrm{dm}^{3} / \mathrm{mol} \cdot \mathrm{s}$, respectively.

\section{Introduction}

Kinetics of metal extraction by chelating agents is important for solvent extraction and liquid membrane operations. Most workers have interpreted their data in terms of aqueous-phase reaction. ${ }^{15,17)}$ For water-insoluble chelating agents, Flett et al..$^{9)}$ and Komazawa et $\mathrm{al}^{16)}$ asserted that the rate determining process would be interfacial in nature. A consistent explanation of the kinetics of metal extraction is lacking at present.

It would be difficult to elucidate the reaction locus of metal extraction based solely on information about the overall extraction rate. A more rational approach is first to determine the mechanism and the rate of metal chelate formation in the aqueous phase and then to study the extraction mechanism on the basis not only of the rate of metal extraction but also the information obtained from the reaction mechanism in the aqueous phase.

Several papers have reported the results of studies on the formation of $1: 1$ metal chelate complexes of various water-soluble $\beta$-diketones, e.g. acetylacetone (Acac) and thenoyltrifluoroacetone (TTA). ${ }^{2,4,13,14,20,21)} \beta$-diketone exists as enol and keto tautomers and the reaction routes to yield $1: 1$ complexes are very complicated.

The aim of the present work is the elucidation of the mechanism and the rate of $1: 1$ complex formation in the aqueous phase for less water-soluble benzoylacetone (BA) by measuring the chemical re-

Received June 21, 1982. Correspondence concerning this article should be addressed to M. Harada. M. Mori is now with Nippon Steel Corp., Tokai, Aichi 476. W. Eguchi is now at Dept. Chem. Eng. Kyoto Univ., Sakyo-ku, Kyoto 606. action rate by use of the relaxation method.

\section{Experimental}

\subsection{Procedures and reagents}

BA aqueous solution and cupric perchlorate aqueous solution were mixed in a chamber using a four-jet mixer and the time course of light absorbance by the solution was measured by a rapid reaction analyzer of stopped-flow type (Union Giken Co. Ltd., PA-1100). The concentration of sodium perchlorate was adjusted to $0.1 \mathrm{~mol} / \mathrm{dm}^{3}$ after the aqueous-solutions were mixed. The $\mathrm{pH}$ values of the aqueous solutions were adjusted to be in the range $2.5-4.5$ using perchloric acid or sodium hydroxide. The reaction temperature was maintained at $25^{\circ} \mathrm{C}$. All reagents used were the same as shown in the previous report. ${ }^{1}$

\subsection{Experimental conditions}

The equilibrium relationships of the reactions in the aqueous phase involving $\mathrm{Cu}$ (II) and $\mathrm{BA}$ have been determined by the authors. ${ }^{1)}$

$$
\begin{aligned}
& \mathrm{HB} \rightleftarrows \mathrm{H}^{+}+\mathrm{B}^{-} ; \\
& K_{2}=[H][B] /[H B]=1.6 \times 10^{-9} \mathrm{~mol} / \mathrm{dm}^{3} \\
& \mathrm{Cu}^{++}+\mathrm{B}^{-} \rightleftarrows \mathrm{CuB}^{+} ; \\
& K_{3}=[C u B] /[C u]\left[[B]=2.0 \times 10^{8} \mathrm{dm}^{3} / \mathrm{mol}\right. \\
& \mathrm{CuB}^{+}+\mathrm{B}^{-} \rightleftarrows \mathrm{CuB}_{2} ; \\
& K_{4}=\left[C u B_{2}\right] /[B][\mathrm{CuB}]=1.6 \times 10^{8} \mathrm{dm}^{3} / \mathrm{mol}
\end{aligned}
$$

Here, HB and $B$ represent neutral and dissociated benzoylacetone. The electrical valences of the ionic species are omitted hereafter for the sake of simplicity. The neutral form of BA consists of the 


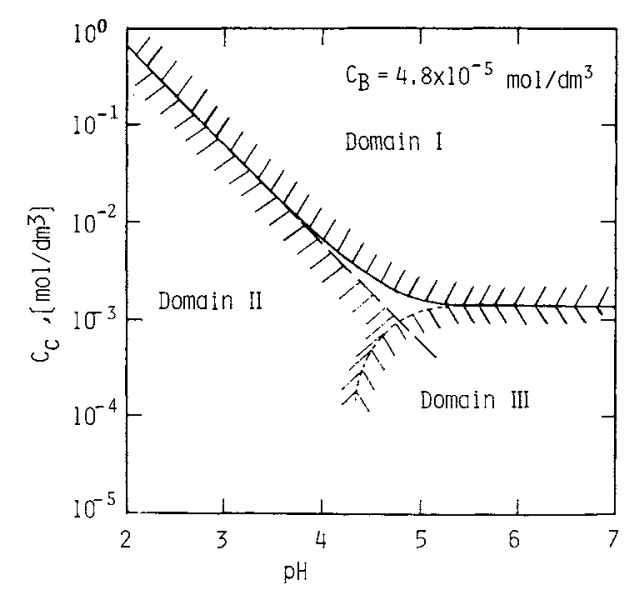

Fig. 1. Experimental domains in this work. Domain $\mathrm{I}, \mathrm{CuB}$ formation; Domain II, coexistence of $\mathrm{CuB}$ and $\mathrm{HB}$; Domain III, $\mathrm{CuB}_{2}$ is not neglected.

tautomers, enol and keto forms. The concentration of $\mathrm{BA},[H B]$, is the sum of those of the two tautomers. The concentrations of the chemical species in the above equations are calculated from the equilibrium relation given in Eqs. (1)-(3). It is easily shown from Eq. (1) that $[B]$ is negligibly small compared with $[H B]$ when the $\mathrm{pH}$ is less than seven.

The condition that $95 \%$ of $\mathrm{BA}$ in the aqueous phase is converted to $\mathrm{CuB}$ complex can be determined from Eqs. (2)-(3) and is shown in Fig. $\mathbf{1}$ as the solid line where the total concentration of $\mathrm{BA}, C_{B}$, is fixed at $4.8 \times 10^{-5} \mathrm{~mol} / \mathrm{dm}^{3}$. The broken curve is the condition that $5 \%$ of $\mathrm{BA}$ remains in the form $\mathrm{HB}$ in the aqueous phase and the dotted curve represents the condition that $5 \%$ of $\mathrm{BA}$ exists in the form $\mathrm{CuB}_{2}$. In domain $\mathrm{I}$ in Fig. 1, the reaction to form $\mathrm{CuB}$ is considered to all intents and purposes to be an irreversible reaction. In domain II, the reaction to form $\mathrm{CuB}$ is considered as a reversible reaction, where $\mathrm{HB}$ is not completely converted to $\mathrm{CuB}$. In domain III, the formation of $\mathrm{CuB}_{2}$ is not negligible. In this experiment, the reaction to form $\mathrm{CuB}$ was carried out in domains I and II.

As shown in the previous report, ${ }^{1)}$ the molar extinction coefficient of $\mathrm{CuB}$ is about 100 times greater than that of $\mathrm{HB}$ at a wavelength of $360 \mathrm{~nm}$, and is about the same value as that for $\mathrm{CuB}_{2}$. When the experiments are performed at the conditions in domains I and II, the absorbance of the solution at $360 \mathrm{~nm}$ is almost completely due to the formation of $\mathrm{CuB}$ except for the early period of the reaction. The absorbance due to $\mathrm{Cu}$ ion is also negligibly small at $360 \mathrm{~nm}$. Therefore, the process to form $\mathrm{CuB}$ can be measured directly.

\section{Results and Discussion}

\subsection{Reaction path}

The tautomerization rate of enol-keto forms of

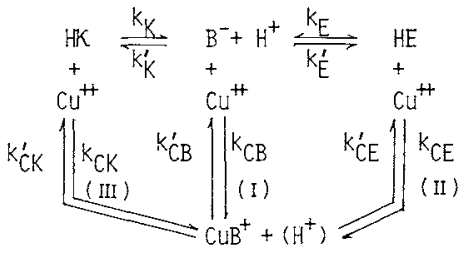

Fig. 2. Postulated reaction paths to yield $\mathrm{CuB}$.

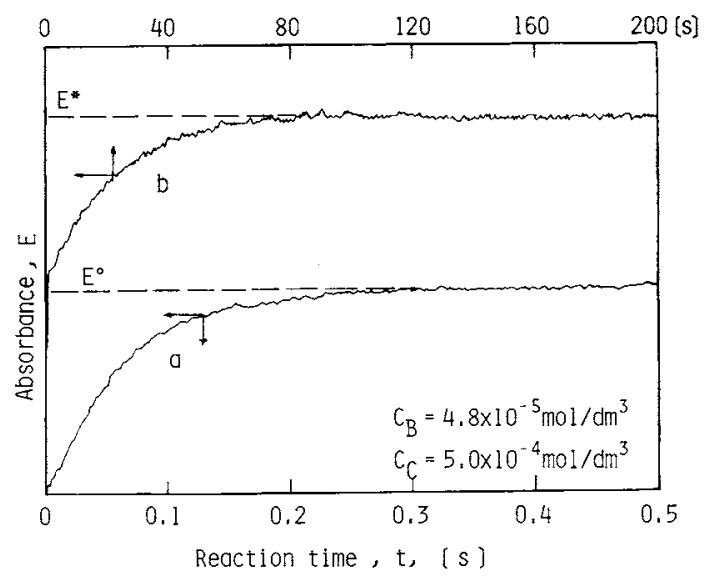

Fig. 3. Typical example of time course of absorbance at initial $\mathrm{pH}: 3.5$.

acetylacetone is expressed in acidic aqueous solution $^{6,12)}$ by

$$
\mathrm{HK} \underset{k_{K}^{\prime}}{\stackrel{k_{K}}{\rightleftarrows}} \mathrm{H}^{+}+\mathrm{B}-\underset{k_{E}}{\stackrel{k_{E}^{\prime}}{\rightleftarrows}} \mathrm{HE}
$$

Here, the rate constants are

$$
\begin{array}{ll}
k_{K}=2.1 \times 10^{-2} \mathrm{~s}^{-1}, & k_{K}{ }^{\prime}=6 \times 10^{6} \mathrm{dm}^{3} / \mathrm{mol} \cdot \mathrm{s} \\
k_{E}=1.4 \times 10^{2} \mathrm{~s}^{-1}, & k_{E}{ }^{\prime}=3 \times 10^{10} \mathrm{dm}^{3} / \mathrm{mol} \cdot \mathrm{s}
\end{array}
$$

HK and HE represent keto and enol form, respectively. Taking this into account, we can postulate the paths of the reaction to form $\mathrm{CuB}$ as shown in Fig. 2.

The chemical reaction to form $\mathrm{CuB}$ via path $\mathrm{I}$ would be very rapid compared with the reactions via paths II and III, because the water coordinated to $\mathrm{Cu}$ ion is easily detached from $\mathrm{Cu}$. Taking into account that the dissociation of $\beta$-diketone in enol form is much faster than for the keto form, the BA in enol form first reacts with $\mathrm{Cu}$ rapidly and approaches quasi-equilibrium. Thereafter, the BA in keto form reacts with $\mathrm{Cu}$ slowly and approaches thermodynamic equilibrium.

To ensure the presence of the above two steps, the reactions to form $\mathrm{CuB}$ were performed at such conditions that the final state of the reactions were in domains I or II in Fig. 1. A typical example of the change of absorbance is shown in Fig. 3 together with the experimental conditions. The ordinate $E$ is the absorbance of the reaction mixture at $360 \mathrm{~nm}$ and the abscissa is reaction time $t$. For a short time span, the 


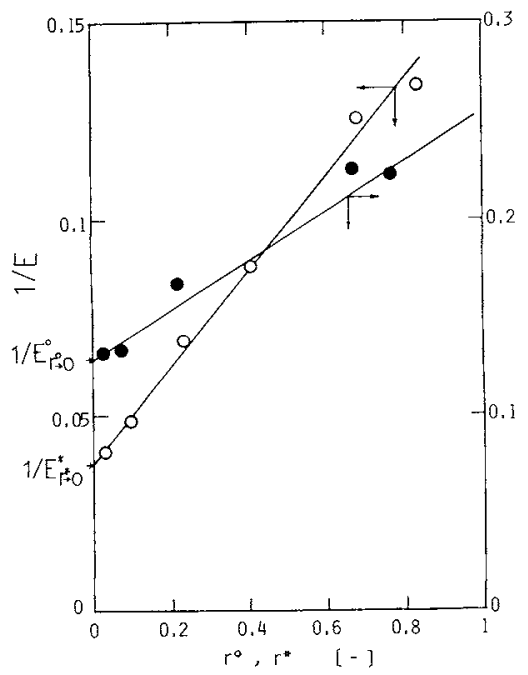

Fig. 4. Enol-keto tautomerization equilibrium.

absorbance rapidly increased with time and attained quasi-equilibrium (curve a). For a large time span, the absorbance initially agreed with the value in the above quasi-equilibrium state (curve b). This experimental result ensures the presence of the two steps in the aqueous-phase reaction. It also ensures that the rate process by intramolecular tautomerization is much slower than the rate processes of Eq. (4).

The quasi- and pure thermodynamic states provide information for the reaction from enol to keto exchange. We can postulate the chemical reactions:

$$
\begin{array}{ll}
\mathrm{HE} \rightleftarrows \mathrm{H}^{+}+\mathrm{B}^{-} ; & K_{2 e}=[H][B] /[H E] \\
\mathrm{HK} \rightleftarrows \mathrm{H}^{+}+\mathrm{B}^{-} ; & K_{2 k}=[H][B] /[H K]
\end{array}
$$

The following relationship exists for the equilibrium constants, $K_{2 e}, K_{2 k}$ and $K_{2}$ :

$$
1 / K_{2}=1 / K_{2 e}+1 / K_{2 k}
$$

We define the tautomerization equilibrium constant $K_{T}$ as

$$
\mathrm{HK} \rightleftarrows \mathrm{HE} ; \quad K_{T}=K_{2 k} / K_{2 e}=[H E] /[H K]
$$

In thermodynamic equilibrium, the following equation is satisfied because $\left[\mathrm{Cu} B_{2}\right]$ is negligibly small in this experiment.

$$
(1 /[C u B])=\left(1 /[C u B]_{r^{*} \rightarrow 0}\right)\left(1+r^{*} / K_{2} K_{3}\right)
$$

Here, $r=[H] /[\mathrm{Cu}]$ and the asterisk represents the value at thermodynamic equilibrium. The above equation is rewritten with use of the absorbances as

$$
\frac{1}{E^{*}}=\left(\frac{1}{E^{*}}\right)_{r^{*} \rightarrow 0}\left(1+\frac{r^{*}}{K_{2} K_{3}}\right)
$$

At quasi-equilibrium Eqs. (5) and (2) hold and these give

$$
\frac{1}{E^{\circ}}=\left(\frac{1}{E^{\circ}}\right)_{r^{\circ} \rightarrow 0}\left(1+\frac{r^{\circ}}{K_{2 e} K_{3}}\right)
$$

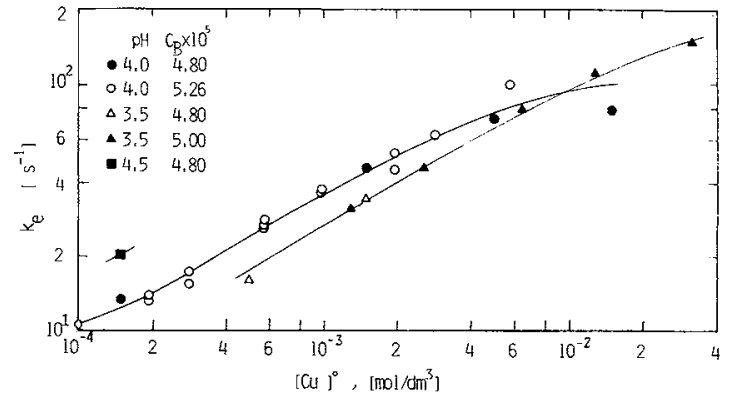

Fig. 5. Observed overall reaction rate coefficients for first step.

where the superscript of open circle represents quasiequilibrium. The absorbances $E^{*}$ and $E^{\circ}$ observed are plotted according to Eqs. (10) and (11) in Fig. 4. The tautomerization equilibrium constant $K_{T}$ can be determined from the intercepts $\left(1 / E^{\circ}\right)_{r^{\circ} \rightarrow 0}$ and $\left(1 / E^{*}\right)_{r^{*} \rightarrow 0}$ and also from the products of the slope of the straight lines, $S$, by $E_{r \rightarrow 0}$ :

$$
K_{T}=\left[E^{\circ} /\left(E^{*}-E^{\circ}\right)\right]_{r^{*}, r^{\circ} \rightarrow 0}=1 /\left[S^{*} E_{r^{*} \rightarrow 0}^{*} / S^{\circ} E_{r^{\circ} \rightarrow 0}^{\circ}-1\right]
$$

The $K_{T}$ value is obtained so that the values evaluated from Eq. (12) agree with each other. The resultant value is given as

$$
K_{T}=0.43
$$

This value compares well with the values 0.52 in water ${ }^{5)}$ and 0.41 in $1 \mathrm{~mol} / \mathrm{dm}^{3}-\mathrm{NaClO}_{4}$ aqeuous solution, ${ }^{23)}$ which were determined from static equilibrium experiments. The agreement of these results of two different techniques ensures that the reaction between $\mathrm{Cu}$ ion and the enol form is much faster than that for the keto form.

\subsection{Reaction mechanism and rates}

Consider first the rate of the reaction to form $\mathrm{CuB}$ from BA in enol form. The measured course of this reaction is given by curve a in Fig. 3. This time course was for a linear process and $\ln \left[\left(E^{\circ}-E\right) / E^{\circ}\right]$ changed linearly with reaction time $t$. In these experiments, the total concentrations of copper $C_{c}$ were much greater than $C_{B}$ and $[\mathrm{Cu}]$ 's were kept nearly constant during the course of the reactions. The $[H]$ values were also kept nearly constant except for the experiment at $\mathrm{pH}=4.5$, where $[H]$ increased by $30 \%$ compared with their initial values. The sole factor changing with time was $[H E]$ as an approximation. The overall reaction rate coefficient $k_{e}$ which is evaluated from the gradient of the straight line of $\ln \left[\left(E^{\circ}-E\right) / E^{\circ}\right]$ against $t$ is shown in Fig. 5.

As mentioned previously, the major path to form $\mathrm{CuB}$ from $\mathrm{HE}$ would be path I in Fig. 2. Since $[B]$ is much less than $[H E]$, a stationary-state method with respect to $\mathrm{B}$ would be usable. In this case, the rate of formation of $\mathrm{CuB}$ is described as 


$$
\begin{aligned}
\mathrm{d}[C u B] / \mathrm{d} t= & k_{C B}[B][C u]-k_{C B}^{\prime}[C u B] \\
& +k_{C E}[H E][C u]-k_{C E}^{\prime}[C u B][H] \\
\mathrm{d}[B] / \mathrm{d} t= & k_{E}[H E]-k_{E}^{\prime}[H][B] \\
& +k_{C B}^{\prime}[C u B]-k_{C B}[B][C u]=0
\end{aligned}
$$

From the above relationships, the rate of $\mathrm{CuB}$ formation is easily expressed when $[\mathrm{CuB}],[\mathrm{HB}] \gg\left[\mathrm{Cu} \mathrm{B}_{2}\right]$, $[B]$ :

$$
\begin{aligned}
& \mathrm{d}[C u B] / \mathrm{d} t=p-k_{e}[C u B] \\
& p=\left\{k_{E} /\left(1+k_{E}{ }^{\prime} r / k_{C B}\right)+k_{C E}[C u]\right\} K_{T} C_{B} /\left(1+K_{T}\right) \\
& k_{e}=\left(1+r / K_{2 e} K_{3}\right)\left\{k_{E} /\left(1+k_{E}{ }^{\prime} r / k_{C B}\right)+k_{C E}[C u]\right\}
\end{aligned}
$$

Equation (15) can easily be solved if $r$ is constant:

$$
\begin{aligned}
\left\{[C u B]^{\circ}-[C u B]\right\} /[C u B]^{\circ} & =\left(E^{\circ}-E\right) / E^{\circ} \\
& =\exp \left(-k_{e} t\right)
\end{aligned}
$$

The measured $k_{e}$ values are replotted according to Eq. (15c) in Fig. 6, where the $k_{E}^{\prime} / k_{C B}$ value was determined to be 13.5 so that the plotted points change linearly, satisfying Eq. (15c). $k_{E}$ and $k_{C E}$ can also be determined from this figure. The other rate constants are obtained with the help of equilibrium constants $K_{2 e}$ and $K_{3}$. The resultant rate constants are

$$
\begin{aligned}
k_{E} & =81 \mathrm{~s}^{-1}, \quad k_{E}{ }^{\prime}=1.5 \times 10^{10} \mathrm{dm}^{3} / \mathrm{mol} \cdot \mathrm{s}, \\
k_{C B} & =1.1 \times 10^{9} \mathrm{dm}^{3} / \mathrm{mol} \cdot \mathrm{s} \\
k_{C B}^{\prime} & =5.7 \mathrm{~s}^{-1}, \quad k_{C E}=2 \times 10^{3} \mathrm{dm}^{3} / \mathrm{mol} \cdot \mathrm{s}, \\
k_{C E}^{\prime} & =2 \times 10^{3} \mathrm{dm}^{3} / \mathrm{mol} \cdot \mathrm{s}
\end{aligned}
$$

Next, we consider the second step of $\mathrm{CuB}$ formation from HK. As mentioned previously, the first step of $\mathrm{CuB}$ formation approached quasi-equilibrium and thereafter the second step of $\mathrm{CuB}$ formation occurred slowly. Thus, quasi-equilibria between B, $\mathrm{CuB}$ and $\mathrm{HE}$ would be attained during whole course of the second step.

$$
[B]=[\mathrm{CuB}] / K_{3}[\mathrm{Cu}]=K_{2 e}[\mathrm{HE}] /[\mathrm{H}]
$$

The change of the sum of $[B],[C u B]$ and $[H E]$ can be described as follows because the formation of $\mathrm{CuB}_{2}$ is negligible in this experiment.

$$
\begin{aligned}
\frac{\mathrm{d}}{\mathrm{d} t}([B]+ & {[H E]+[C u B])=k_{K}[H K]-k_{K}{ }^{\prime}[H][B] } \\
& +k_{C K}[H K][C u]-k_{C K}^{\prime}[C u B][H]
\end{aligned}
$$

The terms $\mathrm{d}[B] / \mathrm{d} t$ and $\mathrm{d}[H E] / \mathrm{d} t$ are related to $\mathrm{d}[\mathrm{CuB}] / \mathrm{d} t$ by differentiating Eq. (18) with respect to time and the introduction of these relations into Eq. (19) yields

$$
\begin{gathered}
\left(1+r / K_{3} K_{2 e}+1 / K_{3}[C u]\right) \mathrm{d}[C u B] / \mathrm{d} t=\left(k_{C K}[C u]+k_{K}\right) \\
\times[H K]-\left(k_{C K}^{\prime}+k_{K}{ }^{\prime} / K_{3}[C u]\right)[H][C u B]
\end{gathered}
$$

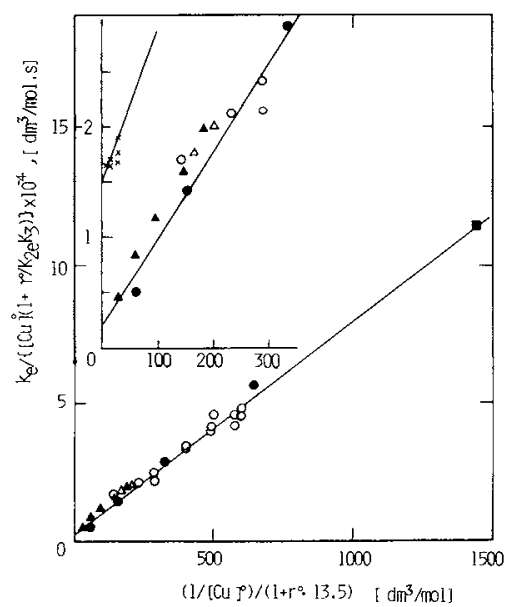

Fig. 6. Validity of Eq. (15c). Symbols are same as in Fig. 5 . Cross symbol represents data by Pearson ${ }^{21)}$ at $25^{\circ} \mathrm{C}$ and ionic strength $0.1 \mathrm{~mol} / \mathrm{dm}^{3}$.

In the above derivation, we assumed that the values $[\mathrm{H}]$ and $[\mathrm{Cu}]$ are constant in the course of the secondstep reaction. Employing Eq. (7) and the relationships

$$
k_{K} / k_{K}{ }^{\prime}=K_{2 k}, \quad k_{C K} / k_{C K}^{\prime}=K_{3} K_{2 k}
$$

the rate of $\mathrm{CuB}$ formation is given from Eq. (20):

$$
\begin{gathered}
\mathrm{d}[\mathrm{CuB}] / \mathrm{d} t=p^{\prime}-k_{k}[\mathrm{CuB}] \\
p^{\prime}=\left(k_{C K}[\mathrm{Cu}]+k_{K}\right) C_{B} /\left(1+1 / K_{3}[C u]+r / K_{3} K_{2 e}\right) \\
k_{k}=\frac{\left(k_{C K}[\mathrm{Cu}]+k_{K}\right)\left(1+1 / K_{3}[\mathrm{Cu}]+r / K_{3} K_{2}\right)}{1+1 / K_{3}[\mathrm{Cu}]+r / K_{3} K_{2 e}}
\end{gathered}
$$

Here, we assumed

$$
C_{B}=[C u B]+[H E]+[H K]+[B]
$$

Integration of Eq. (23) on the postulation that $[\mathrm{Cu}]$ and $[H]$ are independent of time gives

$$
\left(E^{*}-E\right) /\left(E^{*}-E^{\circ}\right)=\exp \left(-k_{k} t\right)
$$

The experimental values of $\ln \left(E^{*}-E\right) /\left(E^{*}-E^{\circ}\right)$ were linear with respect to time and the reaction to form $\mathrm{CuB}$ in the second step is of first order with respect to $\mathrm{BA}$. The values of overall reaction rate coefficient, $k_{k}$, obtained are shown in Fig. 7 where the $r$ value was approximated as $r^{*}$. The result shown in Fig. 7 satisfies the relationship of Eq. (23). From this result the reaction rate constants are determined with help of Eq. (21):

$$
\begin{aligned}
k_{C K} & =11.6 \mathrm{dm}^{3} / \mathrm{mol} \cdot \mathrm{s}, \quad k_{C K}^{\prime}=25.3 \mathrm{dm}^{3} / \mathrm{mol} \cdot \mathrm{s} \\
k_{K} & =0.018 \mathrm{~s}^{-1}, \quad k_{K}{ }^{\prime}=7.86 \times 10^{6} \mathrm{dm}^{3} / \mathrm{mol} \cdot \mathrm{s}
\end{aligned}
$$

Watarai et al. ${ }^{22)}$ determined the rate constant of the tautomerization from enol to keto forms by mixing $\mathrm{BA}$ in dioxane solution with water. The $k_{K}$ value evaluated from their result with use of $K_{T}$ in Eq. (13) is 


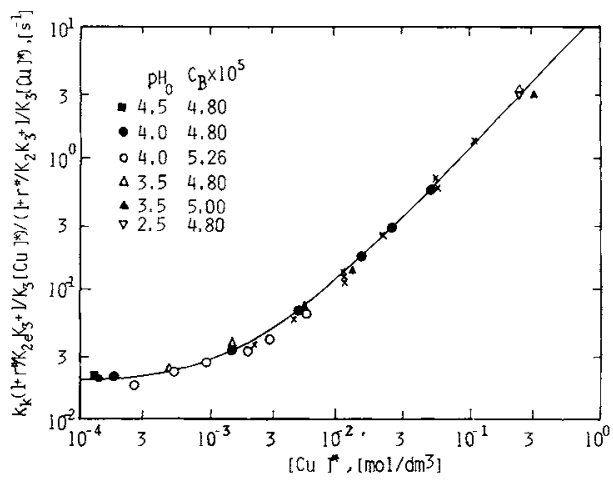

Fig. 7. Overall reaction rate coefficients for second step. Cross symbol represents data by Pearson ${ }^{21)}$ at $25^{\circ} \mathrm{C}$ and ionic strength $0.1 \mathrm{~mol} / \mathrm{dm}^{3}$.

$$
k_{K}=0.025 \mathrm{~s}^{-1}
$$

This value is a little greater than that obtained in this work.

We compare the results of the present work with those of Acac. Pearson et al. ${ }^{21)}$ reported data for the reaction of cupric ion with Acac. They analyzed these data on the assumption that reaction path I in Fig. 2 was neglected. This assumption is not rigorous and we re-analyzed their data according to Eqs. (15c) and (23). The results are shown in Figs. 6 and 7. From these figures, the rate constants can be obtained. The resultant values are shown in Table 1 together with the results of Eigen ${ }^{6)}$ and Munakata et al. ${ }^{18)}$

The $k_{C B}$ values for these two systems agree with each other and this rate process would proceed according to Eigen's mechanism, ${ }^{7}$ which involves the loss of water molecule from the metal ion as the ratedetermining step. The reaction rate constant is given in this case by

$$
k_{C B}=K_{O S} k_{\mathrm{H}_{2} \mathrm{O}}
$$

$K_{O S}$ is the formation constant of the outer sphere complex and is given by Fuoss ${ }^{10)}$ as

$$
\begin{aligned}
K_{O S} & =\left(4 \pi N a^{3} / 3000\right) \exp (-U / k T), \\
U & =-2 e^{2}(1+\kappa a) / D a
\end{aligned}
$$

$N$ is the Avogadro number and the $a$-value is minimum separation distance between ions. The $e$-value represents the elementary electric charge. $U$ represents the electrostatic effect. $D$ and $1 / \kappa$ are the dielectric constant and Debye's screening length, respectively. $k_{\mathbf{H}_{2} \mathrm{O}}$ is the rate constant of dehydration and is given for cupric ion ${ }^{11)}$ as

$$
k_{\mathrm{H}_{2} \mathrm{O}}=3 \times 10^{8} \mathrm{~s}^{-1}
$$

The $k_{C B}$ value calculated from Eq. (27) with use of Eqs. (28) and (29) is given by

$$
k_{C B}=1.4 \times 10^{9} \mathrm{dm}^{3} / \mathrm{mol} \cdot \mathrm{s}
$$

Table 1. Comparison of reaction rate constants for Acac with $\mathrm{BA}$ at $25^{\circ} \mathrm{C}$

\begin{tabular}{lccccc}
\hline Reactions & Acac & Ref. & BA & Ref. \\
\hline $\mathrm{H}^{+}+\mathrm{B}^{-} \rightarrow \mathrm{HE}, k_{E}^{\prime}$ & $3 \times 10^{10}$ & a & $1.5 \times 10^{10}$ & $\mathrm{~d}$ \\
$\mathrm{H}^{+}+\mathrm{B}^{-} \rightarrow \mathrm{HK}, k_{K}^{\prime}$ & $6 \times 10^{6}$ & $\mathrm{a}$ & $7.9 \times 10^{6}$ & $\mathrm{~d}$ \\
$\mathrm{Cu}^{++}+\mathrm{B}^{-} \rightarrow \mathrm{CuB}^{+}, k_{\mathrm{CB}}$ & $9 \times 10^{8}$ & $\mathrm{~b}$ & $1.1 \times 10^{9}$ & $\mathrm{~d}$ \\
$\mathrm{Cu}^{++}+\mathrm{HE} \rightarrow \mathrm{CuB}^{+}, k_{\mathrm{C}}$ & $1.5 \times 10^{4}$ & $\mathrm{c}$ & $2 \times 10^{3}$ & $\mathrm{~d}$ \\
$\mathrm{Cu}^{++}+\mathrm{HK} \rightarrow \mathrm{CuB}^{+}, k_{C K}$ & 11 & $\mathrm{c}$ & 12 & $\mathrm{~d}$ \\
\hline & & & & \\
$\quad$ al. Eigen et al. (1970); d, this work.
\end{tabular}

where we assumed that the $a$-value was $4.8 \times 10^{-10} \mathrm{~m}$ and the ionic strength was $0.1 \mathrm{~mol} / \mathrm{dm}^{3}$. This calculated value agrees well with the experimental result.

The reaction of cupric ion with enol form of $\mathrm{BA}$ is considered to be abnormally slow and this has been interpreted in terms of three factors ${ }^{21)}$ : a) relatively strong intramolecular hydrogen bonds which may convert the protonated ligand into a poor entering group $^{3)}$; b) sterically controlled six-membered chelate ring closure; and c) the energetics of the proton release from half-bonded intermediate which slows down the rate of ring closure.

A pure sterically controlled mechanism predicts that the enolate ion should also react at an abnormally slow rate, and mechanism b) seems to be unreasonable. ${ }^{13)}$ The present result shows that the effect of the substitution from methyl- to phenylgroup on the $k_{C E}$ value would be large. Systematic study of this substitution effect would give information as to the cause of the slow rate, but a detailed discussion is left to future work.

The reaction rate constant $k_{C K}$ is much smaller than $k_{C E}$. The $k_{C K}$ for BA differs little from that for Acac. This reaction with keto form proceeds through an intermediate in which the metal ion is bonded to keto groups. This would be controlled by the slow metalcatalyzed proton release. ${ }^{21)}$

The reaction important for the extraction of metals would be the reaction of metal ions with enol or enolate ionic forms of $\beta$-diketone from the viewpoint of the extraction kintics of metals. At relatively high $\mathrm{pH}$, the reaction of metal ions with enolate ion would be important. At lower $\mathrm{pH}$ and relatively large concentration of metal ions, the direct reaction of metal ion with the enol form would be of primary concern in the kinetics of metal extraction.

\section{Conclusion}

The reaction mechanism of the reactions concerned with cupric ion and benzoylacetone was elucidated and the reaction rates in the aqueous phase were determined. The reaction of cupric ion with enolate ion proceeded according to Eigen's mechanism and 
the reaction rate constant is very large. Direct reactions of cupric ion with molecular enol and keto forms were observed. These rate constants are abnormally small compared with that of the reaction with enolate ion.

\section{Acknowledgment}

The authors gratefully acknowledge the financial support from a Grant-in-Aid for Scientific Research from the Ministry of Education, Science and Culture of Japan.

\section{Nomenclature}

a $\quad=$ minimum separation distance between ions $[\mathrm{m}]$

$C_{B}=$ total concentration of benzoylacetone $\left[\mathrm{mol} / \mathrm{dm}^{3}\right]$

$C_{C} \quad=$ total concentration of copper $\quad\left[\mathrm{mol} / \mathrm{dm}^{3}\right]$

$D \quad=$ dielectric constant [-]

E $\quad=$ absorbance $[-]$

$e \quad=$ elementary electric charge

$K_{i} \quad=$ equilibrium constants for reaction of Eqs. (1)-(3), (5), (6), (8) [in $\mathrm{mol}$ and $\mathrm{dm}^{3}$ units]

$k_{i}=$ reaction rate constants defined in Fig. 2 or in Eq. (29) [in $\mathrm{mol}, \mathrm{dm}^{3}$ and $\mathrm{S}$ units]

$k_{e} \quad=$ reaction rate coefficient for first step of reaction to form $\mathrm{CuB}$

$k_{k} \quad=$ reaction rate coefficient for second step of reaction to form $\mathrm{CuB}$

$N \quad=$ Avogadro number $\quad\left[\mathrm{mol}^{-1}\right]$

$r \quad=[\mathrm{H}] /[\mathrm{Cu}]$

$t \quad=$ time

$U \quad=$ electrostatic effect of outer sphere coordination of ligand with metal ion

[ ] molar concentration

$[\mathrm{J} / \mathrm{mol}]$

$\kappa$

$=$ inverse of Debye's screening length $\left[\mathrm{mol} / \mathrm{dm}^{3}\right]$

$[\mathrm{m}]$

$\langle$ Superscripts〉

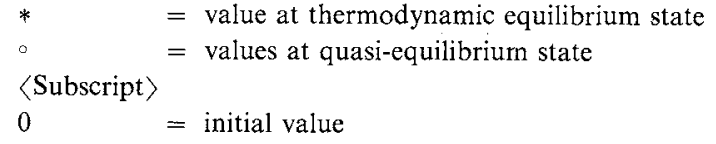

\section{Literature Cited}

1) Adachi, M., H. Yamamoto, M. Harada and W. Eguchi: $J$. Chem. Eng, Japan, 16, 109 (1983).

2) Braril, R. C., M. Cefola, P. S. Gentile and A. V. Celiano: $J$. Phys. Chem., 70, 1358 (1966).

3) Cassatt, J. C. and P. G. Wilkins, J. Am. Chem. Soc, 90, 6045 (1968).

4) Celiano, A. V., M. Cefola and P. S. Gentile: J. Am. Chem. Soc., 65, 2194 (1961).

5) Eidinoff, M. L.: J. Am. Chem. Soc., 67, 2073 (1945).

6) Eigen, M. and L. DeMayer: "Techniques of Organic Chemistry," Vol. VIII, Part II, Interscience Publishers Inc., New York (1963).

7) Eigen, M. and K. Tamm: Z. Elektrochem., 66, 107 (1962).

8) Fay, D. P., A. R. Nichols and N. Sutin: Inorg. Chem., 10, 2096 (1971).

9) Flett, D. S., D. N. Okuhara and D. R. Spink: J. Inorg. Nucl. Chem., 35, 247 (1973).

10) Fuoss, R. M.: J. Am. Chem. Soc., 80, 5059 (1958).

11) Geier, G.: Bunsenges. Physik. Chem., 69, 617 (1965).

12) Hiromi, K.: "Kanwa Gensho no Kagaku," ed. by Azuma, Iwanami Press, Tokyo (1974).

13) Hynes, M. J. and B. D. O'Regan: J.C.S. Dalton, 163 (1979).

14) Jaffe, M. R., D. P. Fay, M. Cefalo and N. Sutin: J. Am. Chem. Soc., 93, 2878 (1971).

15) Kojima, T.: Doctoral thesis, Tokyo Univ. (1981).

16) Komazawa, I., T. Otake and T. Muraoka: J. Chem. Eng. Japan, 13, 204 (1980).

17) Kondo, K., S. Takahashi, T. Tsuneyuki and F. Nakashio: $J$. Chem. Eng. Japan, 11, 193 (1978).

18) Munakata, M. and K. Yamada: Bull. Chem. Soc. Jpn., 51, $3500(1978)$.

19) Nonhebel, D. C.: Tetrahedron, 24, 1869 (1968).

20) Ong, W. K. and H. H. Prince: J. Chem. Soc. Sect A, 458 (1966).

21) Pearson, R. G. and O. P. Anderson: Inorg. Chem., 9, 39 (1970).

22) Watarai, H. and S. Suzuki: Bull. Chem. Soc. Jpn., 50, 757 (1977).

23) Yoshimura, Y. and N. Suzuki: Bull. Chem. Soc. Jpn., 51, 2002 (1978). 